\title{
"It Takes Some Empathy, Sympathy, and Listening": Telephone Outreach to Older Detroiters in a Pandemic as a Modality to Gain an Understanding of Challenges and Resiliency
}

\author{
Vanessa O. Rorai - Tam E. Perry - Sarah E. Whitney - Hannah C. Gianfermi • \\ Jamie A. Mitchell • Kent D. Key • Peter A. Lichtenberg • Robert Joseph Taylor • \\ Joan L. Ilardo • Sean M. Knurek • Christian S. Conyers
}

\begin{abstract}
This manuscript describes a telephone outreach project for members of a research registry program for older adults in Detroit, Michigan. From April until December 2020, the Healthier Black Elders Center designed and implemented a telephone outreach program, calling 1204 older adults utilizing 15 staff and volunteers. The calls served to check in on registry members and collect data on mental health, coping mechanisms, access to services, masks, testing, and tele-health. This paper details the methods of developing and implementing an innovative engagement program that collected time-sensitive data from older Black adults that has directly been applied to create virtual health education programs, share resource information, and create a program to reduce social isolation.
\end{abstract}

V. O. Rorai $(\bowtie)$

Institute of Gerontology, Wayne State University, Detroit, MI, USA e-mail: vrorai@wayne.edu

T. E. Perry $\cdot$ S. E. Whitney $\cdot$ H. C. Gianfermi

School of Social Work, Wayne State University, Detroit, USA

J. A. Mitchell • C. S. Conyers

School of Social Work, University of Michigan, Ann Arbor, USA

K. D. Key

College of Human Medicine, Michigan State University, East Lansing, USA
Keywords Community engagement · CBPR .

Recruitment · Tele-health - Social isolation intervention . Covid-19 · Older adults · African American · Minority aging $\cdot$ Retention

\section{Introduction}

The grave impact of Covid-19 has created a push for senior service providers and researchers to adapt to this crisis in various ways. Telephone contact is one method the present study used to maintain engagement with older adults in the registry and collect time-sensitive information on their needs and overall well-being during the Covid-19 pandemic. This paper describes the experience of conducting telephone surveys with Black older

\section{P. A. Lichtenberg}

Institute of Gerontology, Psychology, Wayne State University, Detroit, USA

\section{P. A. Lichtenberg · R. J. Taylor}

Program for Research on Black Americans, Institute for Social Research, Social Work, University of Michigan, Ann Arbor, USA

R. J. Taylor · J. L. Ilardo

Research Initiatives, College of Human Medicine, Michigan State University, East Lansing, USA

S. M. Knurek

Michigan State University Extension, East Lansing, USA 
adults in the Detroit area as an innovative mechanism to overcome the digital divide during the first year of the pandemic in order to share strategies and lessons learned.

Covid-19 has disproportionately affected African Americans in both contracting and dying from the virus. Across the lifespan when compared to White nonHispanic populations, African Americans are 1.1 times more likely to contract the virus, 2.9 times more likely to be hospitalized, and 1.9 times more likely to die from Covid-19 [1]. Over $75 \%$ of the City of Detroit's residents identify as Black or African American. Within the city, $55.8 \%$ of confirmed Covid-19 cases are among African Americans, and $81 \%$ of the deaths are among Black Detroiters. When looking at age, $13.6 \%$ of the Detroit population is aged 65 and older. More than $20 \%$ of all Covid diagnoses within the city are among those aged 60 and older, and $78.9 \%$ of deaths were those aged 60 and older [2,3].

Segments of these populations are particularly vulnerable to the pandemic's effects as a result of the digital divide. Access to the Internet is not available for everyone. Generally, the number of older adults across the country using the Internet and smartphones has increased each year; however, there is a strong difference in usage among cohorts of older adults. The rate of using the Internet and owning a smartphone is double for older adults aged 65 and younger compared to those over age 70 . This gap is further widened with significantly less access among older adults at an annual household income of $\$ 30,000$ or less and those with less than a college graduate education level [4-6]. Almost 30\% of households in Detroit do not have any broadband Internet access. This number increases to two-thirds for low-income households [7]. Factors such as costs, skills, and low connectivity have contributed to internet access barriers [7]. At least 1 in 5 Detroit households lack broadband subscriptions of any type (including cellular data plans); Detroit ranks as the 19th worst connected city in the USA [8].

Lack of access to the Internet may increase older adults' vulnerability. Previous research has shown "older adults who did not use the Internet or email tended to be older, of racial/ethnic minority status, had less education, worse physical and functional health, fewer social and financial resources, and a greater loneliness/perceived social isolation" [10]. Technology utilization has been proven to impact health outcomes, increase interaction with social ties, and enable access to resources [11-21]. While adoption of various technologies has been determined to be lower for older adults, studies illustrate that they were more inclined to utilize technology if it was perceived to improve their quality of life and increase social connectedness [19, 20].

Telephone outreach programs are used by social service organizations to reduce the impacts of the digital divide. They serve to decrease social isolation and are used to gather timely information on the needs of older adults during a health emergency [22, 23]. They also serve to replace in-person events that are typical in many communities for seniors. Although the use of telephone outreach is not novel, it can contribute to strengthening and increasing the size of outreach to senior populations since it is a readily available technology, does not require learning new skills, and is relatively low cost. For older Black adults involved with research institutions, these types of programs can promote connection or retention to a research context despite the drastic shifts that study participants experience.

\section{Health Risks of Social Isolation}

Literature has well established the detrimental effects social isolation and loneliness can have on older adult's physical and mental health, including shortening lifespan [24-26]. For instance, research among African Americans has found that social isolation is associated with higher rates of depressive symptoms, psychological distress, and serious psychiatric disorders [27, 28]. Prior to Covid-19, the number of isolated older adults was significant at $17 \%$, and a staggering $43 \%$ of older adults experienced loneliness [20, 24]. Upon the declaration of the pandemic, Covid-19 social distancing and public health restrictions became imperative to reduce exposure to the virus. However, researchers are now recognizing the Covid-19 paradox that decreasing human contact to mitigate the spread of Covid-19 increased social isolation.

\section{Survey Research to Engage Older Adults}

The roots of surveying can be traced back centuries through census taking; however, the modern concepts of sampling, questionnaire design, and data collection emerged around 1935 to solidify its place in the social sciences [29]. Surveys established a way to obtain public opinion and collect timely information. Despite 
current acknowledgments of challenges (many people do not have landline telephones, few people answer unknown numbers), some persons, including older adults, may still have high participation in telephone surveys as a means to collect data. Research studies illustrate that telephone surveys have been used to measure health outcomes of older adults [11,30, 31]. Consequently, telephone outreach methods have been incorporated to assess the effectiveness of telemedicine [12]. In addition to assessments, health services such as cognitive behavioral therapy, peer support, and physical health evaluations have been positively received by older participants who were unable to access in-person services $[12,13]$. Thus, the telephone as a modality has many possibilities to support the health and social needs of older adults.

Telephone outreach programs implemented throughout the USA have aimed to reduce the impact of social isolation among older adults during Covid-19. For example, two medical schools in Connecticut and Illinois partnered student volunteers with isolated older adults via phone calls to assess needs, share resources, and engage the call recipients in conversation. Both volunteers and older adults within these programs expressed the profound impact the intergenerational connection had on their life [14]. One innovative program in Los Angeles matches retired community members and volunteers via telephone to increase social connection. This telephone program found great success in increasing healthy social connections and forming a community coalition [15]. Telephone outreach programs have the potential to offer companionship during the pandemic and ameliorate the effects of social isolation.

The current study builds on previous telephone outreach projects in a few ways. The target participants included those most vulnerable during the pandemic based on demographics and geographic location. Considering health disparities experienced by this community, as well as the impact that the Covid-19 pandemic had on the African American population, and the state of Michigan, deeper insights through research in this community contribute new and valuable data to the current literature. It also served multiple purposes in one phone call: to collect a broad scope of data on mental and physical well-being, access to services, and strengths and challenges dealing with the pandemic; to provide resources to address unmet needs; and to utilize this data to tailor program activities. This project displays what can be accomplished without a "matching" system of pairing callers with participants, which will lend to the literature on how results can be achieved with a small research team and large participant pool.

Innovative Approach to Retain Individuals in Research Engagement

Similar to engaging community groups of older adults, telephone outreach could also serve to engage older adults interested in contributing to research. Research registries have been an essential component of research engagement for those historically marginalized by research [32-38]. Research registrants are individuals who agree to be formally entered into a registry aimed to participate in research studies. For some research registries, high contact with registry members is an important part of funded programming. During the pandemic, continued outreach to older Black adults from an academic institution addresses the immediate needs described above (social isolation and timely information) and may reduce attrition of registry members through this increased connection in the years to come. Given the multi-pronged efforts for retaining members prior to Covid-19 (print newsletters, health programming), attention to well-being in a pandemic that disproportionately affects older Black adults can be viewed as an ethical approach to work with older adults and may impact their views of researchers and research institutions for decades to come.

This paper describing a research institution's outreach to older adults has two aims: (1) to describe the development and administration of a telephone outreach program to engage older adults who had already been part of a research registry in a public health crisis and (2) to present key outcomes and lessons learned to contribute to advancing literature and support program replication.

\section{Methods}

All participants who were included in the telephone outreach program were members of the Healthier Black Elders Center, pseudonym (HBEC). Created in 1997, HBEC is funded by the National Institutes of Health and is part of a network of centers called the Resource Centers for Minority Aging Research, pseudonym 
(RCMAR) [39]. Each center within RCMAR focuses on a particular population and/or health condition (specifically Alzheimer's and related dementias). HBEC is the community component of the RCMAR in Michigan, called the Michigan Center for Urban African American Aging Research, pseudonym (MCUAAAR) [32]. HBEC aims to address and reduce health disparities through research and education [32-38]. HBEC maintains a research registry, the Participant Resource Pool, pseudonym (PRP), that consists of approximately 1,200 older Black adults aged 55 and older that mostly live within the Detroit area of Michigan. HBEC has a Community Advisory Board (CAB) of fifteen older Black adults from the Detroit community. The $\mathrm{CAB}$ functions as the program leaders and gatekeepers of research studies that use the PRP for participant recruitment [32]. HBEC typically utilizes multiple methods of outreach to members of the PRP including a bi-annual newsletter, in-person health education events throughout the year, birthday cards, and updates information for all members every 18 months. Figure 1 depicts the logic model created at the inception of the HBEC program. It illustrates the recruitment process for building the research registry that continues to guide and expand HBEC program activities to date including the current telephone outreach project.

Due to the abrupt cancelation of all in-person health education events and research studies, the HBEC staff, $\mathrm{CAB}$, and program leaders decided to develop this telephone outreach project to stay engaged with registry members during the pandemic. This section will describe the resources used to launch and sustain the project and process of conducting the project until completion.

\section{Resources for Launching Project: Caller Team}

The telephone outreach team collectively developed the survey and telephoned participants. First, the project idea was shared with all $\mathrm{CAB}$ members for feedback, and those who were interested and available volunteered to join the project team. All CAB members received continuous project updates at the quarterly board meetings and provided feedback to the project leaders. Due to the abrupt restrictions of in-person activities, many students and research staff had to pause or cancel their planned projects. This resulted in students from various departments joining the project team to complete their independent project or field placement requirements. There were research assistants from other research labs that joined the project team since they could not continue with their own in-person studies. In total, the team comprised of five HBEC Community Advisory Board members, three Wayne State University students from the School of Social Work, one student from the Medical School, one student from the Humanities Center, two research assistants and three staff from the Institute of Gerontology.

This larger program is funded by the National Institutes of Health under its Resource Centers for Minority Aging Research program, specifically its Community

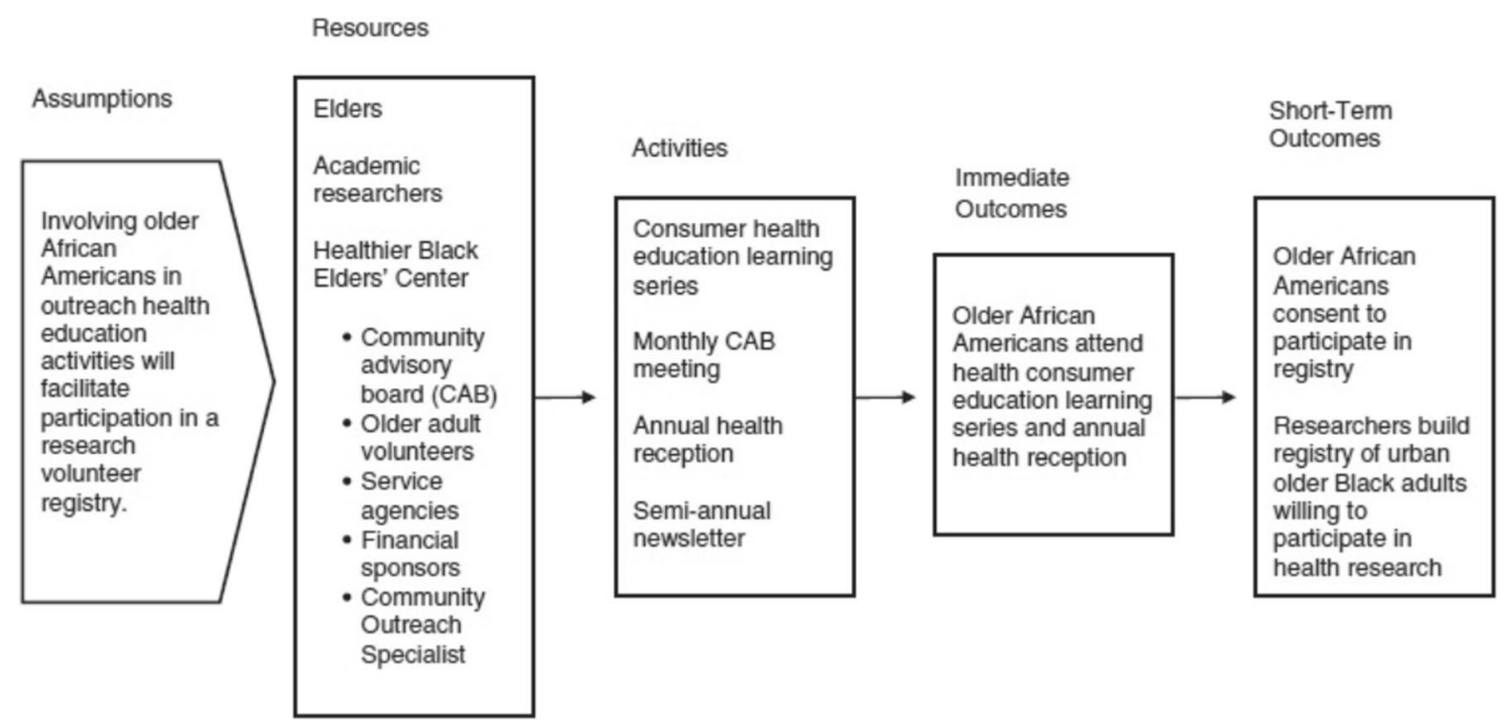

Fig. 1 Logic model illustrating the recruitment process for building the HBEC registry 
Liaison and Recruitment Core (CLRC). To promote "cross-site engagement and learning," the HBEC shared their ongoing progress on this work with the other CLRC's around the country [39]. The telephone outreach program lines up with this core's central mission.

\section{Caller Training}

Prior to beginning the telephone calls, each team member, or callers, completed the required research ethics training, an orientation to the HBEC program, and a training on the survey, telephone script, and data entry. The HBEC program coordinator and lead of the telephone outreach project provided the orientation and training. The training involved practicing administering the survey questions, reviewing the format of contact lists, discussion of strategies for note taking participant answers, and demonstrating how to complete the data entry using the online survey.

Callers were equipped with an extensive directory of organizations and services that they could recommend to participants as resources to address expressed needs. These directories listed resources in categories of needs such as mental health services, food services, utilities, and transportation. Some resource lists were created by state representatives, by the university's social work department, by senior-focused partner organizations, and from the telephone outreach team. Services listed within the resource lists were vetted or known to the HBEC program prior to the telephone outreach project. Callers were trained to use these resource lists as needed based on participant's stated needs.

\section{Survey Design and Data Collection}

The survey instrument was approved by the Institutional Review Board of Wayne State University as part of a fasttrack process for Covid-19-focused studies (see Supplementary materials Appendix 1). To promote rigor in survey design, data collection, and data analysis, these approaches were used: each caller was given a list of persons to contact following agreed upon guidelines (e.g., attempt to contact each participant at least twice between 8 a.m. and 7 p.m.) using a survey script containing set questions. Feedback from callers was incorporated as well as the content of the calls during debrief meetings. Older adults' input was included at every stage, which promotes rigor by providing opportunity for community members to ask questions. Participant compensation was a monthly drawing for two \$50 VISA gift cards among the participants that completed a survey within that month.

Callers began administering the survey in April of 2020. The survey calls started with a wellness check-in question, (e.g., How are you doing?) and proceeded with five categories of survey script items: strengths and challenges (e.g., What has helped you cope the best during this time?); access to services and healthcare (e.g., If tele-health is available, do you have any hesitation about using telehealth?); family and social life (e.g., How many relatives do you see or hear from at least once a month?); mental health (e.g., Please tell me how many days you have felt depressed in the last 30 days.); and access to technology (e.g., Do you regularly use any of the following: Internet on a cell phone, tablet, email, video conferencing?). At the end of the survey, callers were able to provide qualitative reflections on their conversation. The survey also included three scales: the Three-Item Loneliness Scale, standardized abbreviated version Lubben Social Network Scale (LSNS6), and the UCLA Loneliness Scale (version 3) [40-42].

After administering the survey for a month, the call script was amended based on feedback from the project team callers. New questions were added to capture pertinent data or to formalize the capturing of data that was found to be commonly observed by callers. Edits to the script also included rewording of a survey item for clarity. Questions were added on (1) access to healthcare, (2) access to food/supplies, (3) access to technology, and (4) Covid-19 testing. In September 2020, the survey was updated to include questions on grief and loss and outlook on the future. Participant responses were recorded by callers through note taking and entered into a Qualtrics online survey database, including length of conversation in minutes and the callers' general impressions from the conversation.

Toward the end of the survey script, callers shared HBEC program information to update participants on virtual programming. At any point during the call, if a participant asked for information or resources, callers were trained to review the resource lists to provide information for appropriate services that may address that need. If a participant did not explicitly ask for resource information but identified having challenges, callers were trained to ask the participant if they would like resource information to address that need and only provide the resource information when participant answered yes. If an appropriate resource was not included within the resource lists, the caller would inform the participant they would ask the project team leader for an appropriate resource and call the 
participant back with information. If this occurred, that new resource would be added to the resource lists and shared with all team callers. Shared resource information was included in the data entry by callers.

\section{Caller Debrief Meetings}

A critical component to the telephone outreach project was the monthly caller debrief meetings. These virtual meetings were held at the end of each month throughout the study. In these meetings, the research team would discuss some of their findings and challenges and, if necessary, propose changes to the survey script. These meetings also increased methodological rigor as peer support/debriefing is a noted way to assure rigor during the data collection phase. Most importantly, the debrief meetings served as a wellness check-in for the callers themselves, and to discuss as a group any difficult conversations or unexpected challenges. Conducting a project to document participant's experience of Covid-19 while simultaneously living the pandemic themselves can be challenging; thus, the meetings were designed in part to address concerns of potential secondary trauma stress (i.e., indirect exposure to different types of traumatic material) and burnout experienced by callers [43]. In addition to group discussion in debrief meetings, callers were encouraged to contact project leaders (trained social workers and psychologist) at any time for emotional support, troubleshooting, or any concerns. Callers were also trained to immediately contact project leaders if a participant identified any serious safety concerns or issues; however, none occurred throughout the project. The discussions in these meetings, and informal feedback, as well as the planning for the program are highlighted in the findings below. This project had two significant outcomes: (1) understanding the experiences of older Black adults in real time, and (2) understanding the impact of outreach programming on the caller team.

\section{Sample}

Of the 1,242 HBEC members that were contacted, 557 surveys were completed with 89 participants given resource referral information. The average length of call was 26 minutes. Among those that did not participate, 109 had a disconnected phone number, 12 declined due to health reasons, 3 declined due to moving, 4 declined due to recent family death, and 43 were uninterested in participating. Four hundred eleven registry members were left a message but did not return the call, and 110 were unable to leave a message.

All participants identified as African American. However, of the 557 participants, 18 surveys contained incomplete demographics, due to data entry omissions. Thus, the analytic sample for this analysis is 539. The ages of these participants ranged from 57 to 101 , with 77.49 being the average age. The majority were female $(n=489)$, retired $(n=495)$, unmarried $(n=427)$, and had 1 year or more of college education $(n=423)$.

\section{Outcomes}

Understanding the Experiences of Older Black Adults in Real Time

The telephone outreach program yielded important information for understanding the health and social concerns of older African Americans in the context of the Covid-19 pandemic. The calls served to not only collect timely information, but provide vital resources, and input for targeted program planning, and promote continued connection to the organization.

Providing Vital Resources Of the 557 participants, many reported significant changes in lifestyle, emotional challenges, and health concerns; these findings will be reported in future papers. As participants identified a need, callers were trained to use the regularly updated resource lists to provide appropriate contact information for services or support. All participants that identified a need $(n=89)$ were immediately given appropriate resource information during the survey call or during a follow-up call. The most frequently shared resource information was to senior service providers $(n=17)$ that offer extensive programs and services to address multiple needs such as the Area Agency on Aging. Next, participants received HBEC event information and programs to attend $(n=16)$, contact information to financial wellness programs $(n=12)$, home repair and utility support programs $(n=9)$, mental health services $(n=7)$, grocery delivery and food services $(n=5)$, and contact information for medical/Covid-19 services ( $n=3$ ). Participants that could not access personal protective equipment, more relevant in the beginning of the pandemic, were mailed face masks and gloves to their home 
$(\mathrm{n}=10)$. In addition, ten participants were identified by callers in need of a follow-up wellness call to check in on the mental health and well-being of the participant.

Input for Program Planning The next outcome reflects the impact on the HBEC programming. While answering survey questions, many participants identified and shared feelings of grief and loss, challenges avoiding frequent shopping trips, and confusion between Covid-19 and the flu. This input was directly implemented into HBEC programming by tailoring the fall 2020 "Lunch and Learn" series to address these topics. The first fall "Lunch and Learn" was on stocking a smarter pantry that provided grocery shopping strategies to limit exposure during public shopping trips and food safety tips. The second "Lunch and Learn" was on grief and loss during the pandemic by two psychologists who shared information on the signs of grief and depression, coping strategies, treatment options, and available resources. The third "Lunch and Learn" was a question and answer forum with a local family practitioner and a local infectious disease specialist to share timely information on the difference between Covid-19 and the flu.

Furthermore, feedback from the caller team and participant survey answers resulted in tailoring the HBEC newsletter materials. Typically, a bi-annual newsletter is mailed to all HBEC members. In response to the needs and challenges shared in survey responses, an additional newsletter issue was mailed in the summer that included a full-page step-by-step guide with images on how to join a Zoom meeting by phone or computer. The caller team frequently shared participants' limited access to computers and unfamiliarity with Zoom, thus it was critical to include a dial-in option to "Lunch and Learn" events in addition to mailing all program members Zoom instructions. Many participants later shared with callers they used the guide to participate in other Zoom meetings with family, church groups, and other virtual events. A bonus holiday letter, sponsored by the local AARP, was also mailed to all HBEC members that included a resource list and CDC recommended health safety practices during the holidays. The resource list addressed the common needs identified by participants (mental health services, Covid-19 testing centers, food assistance programs, utility, and financial services). Health safety practices were illustrated in a bingo-style table with images and text interspersed to draw readers' attention toward the guidelines (see Supplementary materials Appendix Fig. 2).
Lastly, new programming has been added in the form of a weekly social group that expands this work beyond HBEC members to the larger community. The group is called the "Party Line," and covers a variety of topics that are collectively chosen by participants and facilitators. Topics to date have included preparing for the holidays during a pandemic, mental health, mindfulness, personal histories shared through music and historical events, and a host of other subjects that strike a balance between informative and casual. The group reduces social isolation through participants joining the weekly session either by video or phone and engaging in icebreaker activities, and a brief presentation by facilitators. Conversations are encouraged which effectively puts participants in control of the group and reduces isolation.

Connection to the HBEC Program Typically, HBEC hosts multiple in-person events throughout the year to stay connected with program members. The telephone outreach calls provided an opportunity to touch-base with members when in-person events were not feasible. These calls also provided opportunities to inform program members of virtual "Lunch and Learn" events and add them to an email listserv to receive program information. The oneon-one conversations allowed the callers to let participants know HBEC CAB members, staff, and faculty were thinking of them during the difficult time of the pandemic, valued their continued participation in the program, and served as an information resource. As HBEC plans for post-Covid programming, these connections during the pandemic should pave the way for continuing connection.

Understanding the Impact of Outreach Programming on the Caller Team

In addition to understanding our participants' experiences, it was imperative to understand the impact on the callers themselves. Caller feedback was solicited during monthly debrief meetings in which three major themes emerged: seriousness of the project, communication strategies, and meaningful reflections.

Gravity of the Telephone Outreach Project Throughout the project debrief meetings, callers frequently discussed both the seriousness of participant's health issues and the importance of their role as a caller. This caller, an older adult staff member, described participant's health concerns beyond Covid-19: "Oh yeah you hear about [the second wave] a lot. They're afraid. All of my calls today they were 
afraid of it. And then they go into their health issue - 'I got heart problems... I'm older... I'm only coming out once or twice a week and I do wear my mask...' They are all afraid". Many callers identified feeling a great sense of concern for participants' physical and mental health while recognizing the impact of their role as a caller toward the participants. This was described by one caller, an older adult research assistant, as "There are two areas that get to me, that is those seniors that are having mental health problems and those seniors who have lost people to covid, and that results in some very long conversations and lots of empathy. But it's good for them. At the end of the call they are grateful that they had someone to listen to." Callers took their role seriously to complete the survey with participants while reflecting on what could to be done to meet participant needs, as shared by one of the student callers: "Something that I've found while I've been doing the calls, there's a lot of urgency. I need to be proficient, but it is important to slow down and be fully present for as long as they need you within reason. In general, folks with a need, need holistic support." The seriousness of the participant's pandemic experience solidified the importance of the caller's role in the project. Callers felt a genuine sense of concern for the participant's well-being and an obligation to balance data collection with empathy and compassion.

Communication Strategies Various strategies for successful survey completion were shared throughout the debrief meetings. One being a strategy of when to make calls that also serves as a protective strategy to their own well-being as described by this older adult caller: "I never try to do my whole list [of participants] in one day because sometimes some of the discussions can be so intense, you need a mental break to try and digest. But generally, I take 3-4 hours everyday." Many callers discussed the unpredictability of the calls, some participants would be doing fairly well and completed surveys in a positive tone, while other participants were experiencing significant grief and loss. To prepare for this unpredictability, many callers described using active listening strategies. As described by one of the older adult research assistant callers, "I think the most difficult thing is when you think a call is going easily, but then they say something that's on their mind and then they become a little bit more serious. Just be open to what they're saying." Most callers shared similar experiences of actively listening to participant's responses as a way to prepare for potential resource referral, to elicit further responses, and to engage with participants in a meaningful way.
One Community Advisory Board member (CAB) caller shared "I think the thing of it is being cool. I think we take a rather conversational approach to it as if we were talking to the person face to face ... Explain it to the person like you would want it explained to you." Many callers took this conversational approach to communicating with participants that sometimes included sharing their own personal stories to help participants feel comfortable. An older adult caller self-disclosed, "You have to give people a little hope...Almost everybody that I talked to had a strong belief in God....and through God and just hearing someone else has suffered the same kind of things is often helpful to them." In a larger sense of active listening, feedback from callers and participants was directly implemented in survey design. This validated callers and encouraged their continued feedback. A CAB caller stated, "I am forced to admit I am impressed. The script has evolved over the project. It is useful for the people we are contacting. I kind of like to see that, as well. You are listening to your contacts; you're listening to each other." These communication strategies for active listening and a conversational approach helped strengthen the project, enabling surveys to be completed in a relatively short time while still engaging participants in meaningful conversations.

It is important to note, $84 \%$ of participants $(n=468)$ did not identify a need or ask for resource information. Callers reported on conversations with participants that illustrated the resiliency and coping mechanisms by participants. One advisory board member shared, "I find that many of the seniors are adapting to what's going on... They just try to do things safely by wearing their masks. Some people are still going out golfing. Some people go out to party gatherings... some people are even having church. But they're being cautious... Many seniors that I have spoken with seem to be adapting well... even though we all are aware that we have a lot of shortcomings and limitations." This was shared during the August 2020 debrief meeting, when the state of Michigan had just surpassed 100,000 cases of Covid-19 [44]. Thus, participants were already sharing experiences of coping and resiliency five months into the pandemic.

Meaningful Reflections As previously discussed, many callers engaged in meaningful conversations with participants beyond completing the survey questions. Some callers described how these conversations and the outcomes of the project has affected their own lives. An older adult research assistant working on the project 
shared, "That's what makes it so fulfilling to me. On many occasions, I feel like I am helping people and... after our conversation they come away with a little lighter feeling spirit and that does me well. So, I take as much value out of the calls as they do." Another CAB caller shared, "One of the things that I've learned from you guys and from this is that loneliness and isolation have real, clinical, consequences. So, if we can mitigate that in any way, it is very very very useful." Knowing that some participants expressed significant needs, it was imperative to follow up to address these needs. This sense of ethical obligation also ties into the seriousness of the caller's role in the project and connecting with participants. Early in 2021, follow-up was completed with all participants who expressed a need to ensure, where possible, that they did not perceive the outreach was solely conducted for data collection purposes. Many of the participants have longstanding relationships with HBEC staff and faculty. We felt it was imperative to make these calls since the Detroit area was experiencing such extensive pandemic challenges, particularly with regard to older adults. As stated by an older adult caller, "...the worst things you can do for someone is to throw out a resource and they just end up at a dead end...you don't get on a call when you don't feel like you are ready to listen because those people are under tremendous stress."

The frequent meetings were key to offering support and motivation to callers through the duration of the outreach as they addressed the needs of those surveyed and their own needs during the pandemic. Overall, the caller team discussed a genuine feeling and desire to do their best to serve the participants through active listening, being present, and addressing their needs with helpful resources. The callers appreciated and relied on the orientation training and debrief meetings to be prepared as best as possible for their calls.

\section{Discussion}

This telephone outreach project utilized short telephone surveys to collect data on the lived experiences of older adults during the Covid-19 pandemic and recommend resources to older adults who were experiencing challenges. The knowledge gained from this project contributes to our understanding of callers' engagement on outreach projects. This knowledge can directly contribute to the possible replication of this project and similar projects in terms of engaging older adults using a lowtech approach (e.g., telephone) and outreach teams including the training of staff, and volunteers including students to work with older adults in cases where faceto-face and virtual interactions are not feasible.

Feedback from those completing the 557 calls noted how enjoyable the conversations were for both caller and research registry members. Some noted that the in person group health engagement events which are a long-established staple of the HBEC [see 32-38] do not take a dyadic approach, so this created an opportunity for sharing individual stories with each other. The telephone outreach team members appreciated the opportunity to offer help in a Covid-safe approach at such a difficult time. Some Community Advisory Board members, who previously played other roles in HBEC to promote research [32-38] in the programming, felt compelled to stay engaged with Participant Resource Pool members in this way.

As agencies serving older adults mobilized to decrease social isolation and support needs in 2020 during the Covid-19 crisis, the HBEC in Detroit created a program that bridged the digital divide, provided a touch point to reduce isolation, recommended pertinent resources, and gathered a rich data set that documents this historic time. In this paper, the authors documented the framework for a telephone outreach program that involved supporting callers and engaging over 500 participants. During this pandemic, participation of both callers and respondents in the midst of experiencing and witnessing the devastating impact of Covid-19 on older Black adults is acknowledged. The project team was able to provide follow-up for all cases for which it was required. This included recommending pertinent resources for mental health services and food insecurity, and sending masks when respondents indicated difficulty obtaining masks in the early days of the pandemic. The training and debriefing ensured callers understood the importance of follow-up. It was feasible because this telephone outreach program had an assigned staff person (the project lead) who could address identified needs in a thorough manner. Another reason for such a successful program is that HBEC is part of a larger university; so as the university worked to address the needs of the community through various programs, this project was acknowledged and supported in fulfilling some of the larger university outreach goals.

By being part of HBEC research registry group, the respondents were accustomed to participating in activities and receiving mailings. Due to the nature 
of the HBEC participant group, with its attention to research engagement and ongoing presence of researchers at events throughout the years, this group's participation levels are viewed as an indication of comfort with this program for two reasons: (1) they know the organization contacting them and (2) they are comfortable being involved with research conducted by the HBEC. Additionally, the demographic makeup of research registry members has a high education level (most have at least 1 year of college) which may also contribute to their comfort with participating in programs generated from a local research university.

This telephone outreach program reflects HBEC's longstanding commitment to community engagement that is a cornerstone of the over 20 year program. This is shown by the support of MCUAAAR leadership for developing pandemic outreach for HBEC members and the logistics of outreach. The survey design and caller orientation utilized input from older adults (e.g., CAB members), and program staff with experience working with older adults on numerous projects in Detroit that translated their expertise to this program. The information gained through this project led to the development of follow-up approaches for a subgroup of those who received initial calls and further programming (e.g., party line). Perhaps most notable is how high contact with program participants and continued input from them are values to be modeled by institutions of higher education working in community based research modalities. We anticipate strengthened relationships due to the planning and implementation strategies described above in several ways: (1) older adults enrolled in the registry may trust researchers' interest in their health and well-being; (2) CAB members enhanced their skills as leaders through serving as callers and writing newsletter articles (e.g., CAB member's experience of parent dying from Covid-19); (3) partnerships with other community organizations were strengthened (e.g., sponsorship of newsletters, circulation of newsletters beyond PRP participants); (4) staff refined their skills for training graduate students to work with older adults; (5) students who had not considered careers in aging are more exposed to this sector; (6) staff and faculty of HBEC strengthened their working relationships; and (7) faculty from the other MCUAAAR cores and other RCMAR's recognize the importance of the contributions of the Community Liaison and Recruitment Core in building a knowledge base of Black older adults.

\section{Limitations}

There were limitations in the administering the telephone outreach program and the collecting the data. First, the participants may not reflect other older adults given their familiarity with the program and research more broadly. The callers themselves varied in professional experience, educational background, and age, which may have influenced aspects of the conversation they chose to document. To counter these differences, all callers involved were orientated on the HBEC program goals and registry approach.

The authors expect that this expanded use of telephone outreach could be an innovative strategy to address immediate needs of older Black adults and hopefully promote retention for the research registry in the future. These findings can be utilized in terms of public health strategies, programs designed for health information dissemination, and retention of minority older adults in research. As a program funded to engage older Black adults in research, the pandemic broadened our engagement strategies to include supporting those who offer their lived experiences to researchers in our community in a low-tech manner. Sharing experiences between callers and respondents allowed this program to become a site of support and urgently needed representation of those historically marginalized and harmed by research.

Supplementary Information The online version contains supplementary material available at https://doi.org/10.1007/s11524021-00564-9.

Acknowledgements We dedicate this paper to Dr. James S. Jackson, our center's Principal Investigator from its original formation until his death (1997-2020). He strongly supported additional outreach to our registry members during the pandemic including this telephone outreach approach. We are honored and humbled by his contributions to minority aging and remain steadfast. We thank all of the members of the telephone outreach team. We know that their active 
listening and resource guidance was beneficial to our members in this unprecedented moment in history. This study was supported by a grant from the National Institutes of Health, 5P30 AG015281, and the Michigan Center for Urban African American Aging Research.

\section{References}

1. Hospitalization and Death by Race and Ethnicity. Cdc.gov. https://www.cdc.gov/coronavirus/2019-ncov/coviddata/investigations-discovery/hospitalization-death-by-raceethnicity.html. Last updated February 12 2021. Accessed February 132021.

2. Covid-19 Dashboard. Demographic data: Detroitmi.gov. https://codtableau.detroitmi.gov/t/DHD/views/ityofDetroitPublicCOVIDDashboard/DemographicCasesDashboard?\%3 AisGuestRedirectFromVizportal=y\&\%3Aembed=y\&\%3 Amobile=true. Last updated 16 February 2021. Accessed 13 February 2021.

3. Quick facts: https://www.census.gov/quickfacts/fact/table/ detroitcitymichigan,MI/EDU685219. Census.gov. Accessed May 18, 2021.

4. Anderson M and Perrin A. Tech adoption climbs among older adults. Pew Research Center: Internet and Technology https://libguides.usc.edu/c.php?g=293795\&p=1957132. Accessed May 172021

5. Chatters LM, Taylor HO, Taylor RJ. Older Black Americans during COVID-19: race and age double jeopardy. Health Educ Behav. 2020;47(6):855-60. https://doi.org/10.1177 /1090198120965513.

6. Pew Research Center. (2019). Internet/broadband fact sheet. https://www.pewresearch.org/internet/fact-sheet/internetbroadband/. Accessed May 17, 2021.

7. Reisdorf, $\mathrm{B}$ and Hampton, $\mathrm{K}$ and Fernandez, L and Dutton, W H., Broadband to the neighborhood: digital divides in Detroit. Published 16 January 2018. https://doi.org/10.2139 /ssrn.3103457

8. Worst Connected Cities. National Digital Inclusion Alliance. Digitalinclusion.org. https://www.digitalinclusion.org/worstconnected-2018/ Published 2018. Accessed May 17, 2021.

9. Choi NG, Dinitto DM. The digital divide among lowincome homebound older adults: Internet use patterns, eHealth literacy, and attitudes toward computer/Internet use. J Med Internet Res. 2013;15(5):e93. https://doi. org/10.2196/jmir.2645.

10. Mayer CJ, Steinman L, Williams B, Topolski TD, LoGerfo J. Developing a Telephone Assessment of Physical Activity (TAPA) questionnaire for older adults. Prev Chronic Dis. 2008;5(1):A24.

11. Brenes GA, Danhauer SC, Lyles MF, Hogan PE, Miller ME. Telephone-delivered cognitive behavioral therapy and telephone-delivered nondirective supportive therapy for rural older adults with generalized anxiety disorder: a randomized clinical trial. JAMA Psychiatry. 2015;72(10):1012-20. https://doi.org/10.1001/jamapsychiatry.2015.1154.

12. Garg A, Goyal S, Thati R, Thati N. Implementation of telemedicine in a tertiary hospital-based ambulatory practice in detroit during the COVID-19 pandemic: observational study. JMIR Public Health Surveill. 2021;7(1):e21327. https://doi.org/10.2196/21327.

13. Office EE, Rodenstein MS, Merchant TS, Pendergrast TR, Lindquist LA. Reducing social isolation of seniors during COVID-19 through medical student telephone contact. J Am Med Dir Assoc. 2020;21(7):948-50. https://doi.org/10.1016 jj.jamda.2020.06.00.

14. Van Dyck LI, Wilkins KM, Ouellet J, Ouellet GM, Conroy ML. Combating heightened social isolation of nursing home elders: the telephone outreach in the COVID-19 outbreak program. Am J Geriatr Psychiatry. 2020;28(9):989-92. https://doi.org/10.1016/j.jagp.2020.05.02.

15. Corley C, Feldman M, Kaiser S. The Social Isolation Impact Project: Motion Picture \& Television Fund Engages the Industry and Community in Staying Connected. Public Policy \& Aging Report. 2017;27(4):156-7. https://doi. org/10.1093/ppar/prx024.

16. Khosravi P, Rezvani A, Wiewiora A. The impact of technology on older adults' social isolation. Comput Hum Behav. 2016;63:594-603. https://doi.org/10.1016/j. chb.2016.05.092.

17. Luijkx K, Peek S, Wouters E. "Grandma, you should do itit's cool" older adults and the role of family members in their acceptance of technology. Int J Environ Res Public Health. 2015;12(12):15470-85. https://doi.org/10.3390 /ijerph12121499.

18. Wu YH, Damnée S, Kerhervé H, Ware C, Rigaud AS. Bridging the digital divide in older adults: a study from an initiative to inform older adults about new technologies. Clin Interv Aging. 2015;10:193-200. https://doi.org/10.2147 /CIA.S72399.

19. Berkowsky R, Sharit J, Czaja S. Factors predicting decisions about technology adoption among older adults. Innov Aging. 2017;1(3):igy002. https://doi.org/10.1093/geroni/igy002.

20. Barbosa Neves B, Franz R, Judges R, Beermann C, Baecker R. Can digital technology enhance social connectedness among older adults? A feasibility study. J Appl Gerontol. $2019 ; 38(1): 49-72$. https://doi.org/10.1177 /0733464817741369.

21. Schifeling CH, Shanbhag P, Johnson A, Atwater RC, Koljack C, Parnes BL, et al. Disparities in video and telephone visits among older adults during the COVID-19 pandemic: cross-sectional analysis. JMIR Aging. 2020;3(2):e23176. https://doi.org/10.2196/23176.

22. Biese K, Lamantia M, Shofer F, et al. A randomized trial exploring the effect of a telephone call follow-up on care plan compliance among older adults discharged home from the emergency department. Acad Emerg Med. 2014;21(2): 188-95. https://doi.org/10.1111/acem.12308.

23. Berkman P, Heinik J, Rosenthal M, Burke M. Supportive telephone outreach as an interventional strategy for elderly patients in a period of crisis. Soc Work Health Care. 1999;28(4):63-76. https://doi.org/10.1300/J010v28n04_05.

24. Social isolation is more than feeling lonely: AARP Foundation. https://connect2affect.org. Nd. Retrieved June 16, 2020.

25. Cudjoe TKM, Roth DL, Szanton SL, Wolff JL, Boyd CM, Thorpe RJ. The epidemiology of social isolation: National Health and Aging Trends Study. J Gerontol B Psychol Sci 
Soc Sci. 2020;75(1):107-13. https://doi.org/10.1093 /geronb/gby037.

26. Hawkley LC, Cacioppo JT. Loneliness matters: a theoretical and empirical review of consequences and mechanisms. Ann Behav Med. 2010;40(2):218-27. https://doi.org/10.1007 /s12160-010-9210-8.

27. Nguyen AW, Taylor RJ, Taylor HO, Chatters LM. Objective and subjective social isolation and psychiatric disorders among African Americans. Clin Soc Work J. 2020;48:8798. https://doi.org/10.1007/s10615-019-00725-z.

28. Taylor RJ, Taylor HO, Nguyen AW, Chatters LM. Social isolation from family and friends and mental health among African Americans and Black Caribbeans. Am J Orthopsychiatry. 2020;90(4):468-78. https://doi. org/10.1037/ort0000448.

29. Ornstein M. A Companion to Survey Research. London, : SAGE Publications Ltd; 2013. doi:10.4135/9781473913943

30. Conn DK, Madan R, Lam J, Patterson T, Skirten S. Program evaluation of a telepsychiatry service for older adults connecting a university-affiliated geriatric center to a rural psychogeriatric outreach service in Northwest Ontario. Canada Int Psychogeriatr. 2013;25(11):1795-800. https://doi.org/10.1017/S104161021300118X.

31. Vallance JK, Eurich DT, Gardiner PA, Taylor LM, Stevens G, Johnson ST. Utility of telephone survey methods in population-based health studies of older adults: an example from the Alberta Older Adult Health Behavior (ALERT) study. BMC Public Health. 2014;14:486. https://doi. org/10.1186/1471-2458-14-486.

32. Mitchell J, Perry T, Rorai V, Ilardo J, Lichtenberg P, Jackson J. Building and sustaining a community advisory board of African American older adults as the foundation for volunteer research recruitment and retention in health sciences. Ethn Dis 2020;30(Suppl 2):755-64. https://doi. org/10.18865/ed.30.S2.755.

33. Lichtenberg PA. The generalizability of a participant registry for minority health research. Gerontologist 2011;51(Suppl 1):S116-24. https://doi.org/10.1093/geront/gnr021.

34. Hall LN, Ficker LJ, Chadiha LA, Green CR, Jackson JS, Lichtenberg PA. Promoting retention: African American older adults in a research volunteer registry. Gerontol Geriatr Med 2016;2:2333721416677469. https://doi. org/10.1177/23337214166774.

35. Chadiha LA, Washington OG, Lichtenberg PA, Green CR, Daniels KL, Jackson JS. Building a registry of research volunteers among older urban African Americans: recruitment processes and outcomes from a community-based partnership. Gerontologist 2011;51(Suppl 1):S106-15. https://doi.org/10.1093/geront/gnr03.

36. Rorai V, Perry TE. An Innovative telephone outreach program to seniors in Detroit, a City facing dire consequences of COVID-19. J Gerontol Soc Work 2020;63(6-7):713-6. https://doi.org/10.1080/01634372.2020.1793254.

37. Nápoles AM, Chadiha LA, Resource Centers for Minority Aging Research. Advancing the science of recruitment and retention of ethnically diverse populations. Gerontologist 2011;51(Suppl 1):S142-6. https://doi.org/10.1093 /geront/gnr019.

38. Mezuk B, Tarraf W, Johnson-Lawrence V, Ilardo J, Lichtenberg P, Jackson J. Analytic training for junior investigators in minority aging research: The Michigan model. The Gerontologist 2020;60(8):1392-402. https://doi. org/10.1093/geront/gnaa001.

39. Resource Centers for Minority Aging Research (RCMAR). National Institute on Aging. https://www.nia.nih. gov/research/dbsr/resource-centers-minority-agingresearch-rcmar. Accessed on May 17, 2021.

40. Hughes ME, Waite LJ, Hawkley LC, Cacioppo JT. A short scale for measuring loneliness in large surveys: results from two population-based studies. Res Aging. 2004;26(6):65572. https://doi.org/10.1177/0164027504268574.

41. Lubben JE, Gironda MW. Social support networks. In: Osterweil D, Brummel-Smith K, Beck J, editors. Comprehensive geriatric assessment. New York, NY: McGraw-Hill; 2000. p. 121-37.

42. Russell DW. UCLA Loneliness Scale (Version 3): Reliability, validity, and factor structure. J Pers Assess. 1996;66(1):20-40. https://doi.org/10.1207/s15327752 jpa6601_2.

43. Cieslak R, Shoji K, Luszczynska A, Taylor S, Rogala A, Benight CC. Secondary trauma self-efficacy: concept and its measurement. Psychol Assess. 2013;25(3):917-28. https://doi.org/10.1037/a0032687.

44. Shamus, K. (2020). Michigan marks $100 \mathrm{~K}$ coronavirus cases in 5 months. Experts warn we'll see at least $100 \mathrm{~K}$ more. Detroit Free Press. https://www.freep. com/story/news/health/2020/08/16/coronavirus-covid-19cases-100-k/3375267001/. Accessed May 17, 2021.

Publisher's Note Springer Nature remains neutral with regard to jurisdictional claims in published maps and institutional affiliations. 